\title{
Systematic Review with Meta-analysis: Denosumab in Glucocorticoids Induced Osteoporosis
}

\author{
Mariya M El Akkawi ${ }^{1^{*}}$ (D) and Ashish Shrestha ${ }^{2}$ \\ ${ }^{1}$ First Affiliated Hospital of Jinan University, Tian He, Guangzhou, Guangdong, China \\ ${ }^{2}$ Fifth Affiliated Hospital of Sun Yat-sen University, Xiangzhou, Zhuhai, Guangdong, China
}

*Corresponding author: Mariya M El Akkawi, First Affiliated Hospital of Jinan University, Tian He, Guangzhou, Guangdong, China

\begin{abstract}
Background: American College of Rheumatology recommends Bisphosphonates as the first choice drug for Glucocorticoids Induced Osteoporosis (GIOP). But denosumab is the promising drug that is recommended as the first choice for post-menopausal osteoporosis. This study was conducted to light up the efficacy and safety of denosumab compared to bisphosphonates in GIOP.
\end{abstract}

Methods: Randomized Controlled Trials (RCTs), retrospective, prospective observational Studies, conference abstracts were searched in PubMed, Web of Science, Embase, China National Knowledge Infrastructure (CNKI), clinicaltrials.gov, WHO clinical trials registry, Cochrane library. Studies analyzing the use of denosumab for GIOP were identified and reviewed independently by 2 authors for inclusion. A random-effect model was used for meta-analysis and was conducted using Review Manager 5.3.

Results: 12 studies with 1562 patients were identified for qualitative analysis. 5 (out of 12) studies were included for meta-analysis to compare efficacy and safety of denosumab to bisphosphonates. There was no statistically significant difference between denosumab and bisphosphonates groups for the fracture risk: risk ratio $(R R)=0.62,95 \%$ Confidence interval $(\mathrm{Cl})=[0.19,1.99]$; adverse events: $R R$ $=1.67,95 \% \mathrm{Cl}=[0.75,3.75]$ and Change in femoral neck Bone mineral density (BMD): weighted mean difference $(\mathrm{MD})=1.0295 \% \mathrm{Cl}=[-0.76,2.80]$. But there was statistically significant difference in change in total hip BMD and lumbar spine BMD: $\mathrm{MD}=1.0395 \% \mathrm{Cl}=[0.12,1.94]$ and $\mathrm{MD}$ $=2.4895 \% \mathrm{Cl}=[1.74,3.23]$ respectively.

Conclusion: This study suggested that there was no significant difference in adverse events and fracture risk between denosumab and bisphosphonates but there was a rise in BMD in total hip and lumbar spine in the denosumab group. Thus, long-term follow-up might report fracture risk-benefit in favor of denosumab in GIOP. More long-term follow-up RCTs are required to crystallize the safety and complications of denosumab.

\section{Keywords}

Glucocorticoid, Osteoporosis, Denosumab, Bisphosphonates, Meta-analysis, Secondary osteoporosis

\section{Introduction}

Glucocorticoids (GC) are one of the important drugs that are widely used for inflammatory and autoimmune diseases. But it comes with the price of many side effects on various organ systems. One of the side effects being osteoporosis, in fact Glucocorticoids Induced Osteoporosis (GIOP) is the most common cause for secondary osteoporosis [1]. In patients taking long term GC, up to $10 \%$ are diagnosed with clinical fractures and up to $40 \%$ have radiographic evidence of vertebral fracture $[2,3]$. And also, for the same BMD risk of fracture is higher in GIOP than postmenopausal osteoporosis [4]. Locascio, et al. reported that there is a loss of up to $12 \%$ BMD during the first few months of initiating corticosteroids and after continue to decrease up to $4 \%$ annually [5]. Despite all these evidences only a few receive preventive therapy for GIOP and many receive treatment only after fracture [6].

Bisphosphonates are recommended as the first drug of choice for prevention and treatment of GIOP along with optimizing calcium, vitamin $D$ and life style modifications as smoking cessation, limiting alcohol, weight bearing exercise [7]. But patients taking long 
term GC also include children, young men and premenopausal women. Safety of use of bisphosphonates in these groups of population is not well understood $[8,9]$ and drug compliance with bisphosphonates is poor [10] which makes this study a necessary, to find out if denosumab can be the alternative for prevention and treatment of GIOP.

Denosumab is a fully human monoclonal (igG2) antibody which attaches to Receptor activator of nuclear factor-KB ligand (RANKL) inhibiting interaction with RANK on the osteoclast membrane thus blocking differentiation, activation and survival of osteoclast. This result decrease in resorption of bone to the formation of bone, leading an increase in BMD and reducing fracture risk. Several RCTs and studies have shown the efficacy and safety of denosumab compared to bisphosphonates in post-menopausal osteoporosis [11-14]. American Association of Clinical Endocrinologists and American College of Endocrinology Clinical Practice guidelines recommends the use of denosumab as the first line for treatment and prevention of postmenopausal osteoporosis [15]. But pathophysiology of post-menopausal osteoporosis and GIOP is different. In post-menopausal osteoporosis, there is increased resorption of bone via RAN$\mathrm{KL}$ due to estrogen deficiency $[16,17]$ whereas in GIOP there is not only increased resorption of bone due to $\mathrm{GC}$ but also there is antagonist effect of GC to Wnt signaling which causes inhibition of osteoblast differentiation $[18,19]$. GC also reduces absorption of calcium from the intestine and increase excretion from the kidney.

Due to above mentioned drawbacks of bisphosphonates in GIOP, established efficacy of denosumab to treat primary osteoporosis and also rapid reversibility of effect of denosumab after discontinuation [20] could make denosumab a better choice of drug for young population particularly in premenopausal women with childbearing potential. It has been approved to use in GIOP only in men and women with a) Multiple risk factors for fracture b) History of osteoporotic fracture c) Who have failed or are intolerant to other anti-osteoporotic treatments d) Who are either continuing or initiating $\geq 7.5 \mathrm{mg} /$ day prednisolone or its equivalent and planned to use the drug for at least 6 months [21]. Thus, this study was conducted to analyze current knowledge on efficacy and safety of denosumab in GIOP.

\section{Methods}

This study was conducted according to Preferred reporting items for systematic reviews and meta-analyses: The PRISMA statement [22]. Search strategy, inclusion criteria, exclusion criteria, data extraction, method of bias assessment, statistical analysis were determined prior to the start of the study.

\section{Search strategy}

Electronic database of PubMed, Web of Science, Embase, China National Knowledge Infrastructure (CNKI), clinicaltrials.gov, WHO clinical trials registry, Cochrane library were searched from inception to January 25, 2019 using keywords and corresponding Mesh terms of "denosumab", denosumab bisphosphonate "glucocorticoid induced osteoporosis", "steroid induced osteoporosis", "glucocorticoid" and "osteoporosis". References of selected studies were manually searched for additional studies. All references were imported to EndNote X9.

\section{Study eligibility}

Studies with following criteria were included 1) Population: Patients taking GC or diagnosed as GIOP 2) Intervention: Denosumab (at any dose and duration) used to treat or prevent GIOP given subcutaneously 3) Comparison: Comparison with bisphosphonates or placebo or baseline data 4) Outcomes: With any of following outcomes: Fracture risk, adverse events, change in bone turnover markers, change in BMD in total hip, lumbar spine, femoral neck, distal radius, greater trochanter 5) RCTs, retrospective, prospective observational Studies, conference abstracts 6) Written in any languages. Studies would be excluded if 1) Post-menopausal osteoporosis patients on steroids treated with denosumab 2) Cancer patients 3) GIOP treated with teriparatide or other medications than denosumab.

Based on the above criteria two authors independently screened for possible studies and classified as included and unclear. Unclear studies were discussed with the third author (SX) and were decided for inclusion. Studies were included based on study titles first then duplicates were removed using EndNote X9. Full abstracts were then read and studies were selected for full article reading.

\section{Data extraction}

Two authors independently extracted data from selected studies. Study name, study design, mean age, mean prednisolone dose during initiation of denosumab, mean prednisolone duration, intervention used and number of participants, co interventions, duration of study, outcomes of study were extracted. Outcomes on fracture risk, adverse events, change in BMD in total hip, lumbar spine, femoral neck, distal radius, greater trochanter were extracted quantitatively. Authors were not contacted for missing data.

\section{Bias assessment}

Two authors independently assessed bias in included studies based on Cochrane collaboration's tool for assessing risk of bias [23]. The tools included were random sequence generation, allocation concealment, blinding and selective outcome reporting. Studies were considered with high risk of bias if randomization was absent, participants were not blinded to treatment and more than 2 included tools had high risk of bias. Unclear bias was considered if more than 2 included tools had unclear risk of bias. 


\section{Statistical analysis}

Review manager 5.3 was used to conduct meta-analysis. Random effects model was used for obvious presence of between study heterogeneity. Dichotomous data were expressed as risk ratio (RR) with 95\% confidence interval $(\mathrm{Cl})$. Continuous data were expressed as weighted mean difference (MD) with $95 \% \mathrm{Cl}$. Funnel plot was used to assessed the publication bias. In Sensitivity analysis, meta-analysis was done removing each study at a time. Sub-group analysis was done on basis of risk of bias: high risk and unclear risk. Heterogeneity was evaluated using $\mathrm{I}^{2}$ tests.

\section{Results}

\section{Search results}

After the search, 354 relevant articles were found and imported to EndNote version X9. After removing duplicates 267 articles were screened on basis of titles and abstracts, 23 articles were assessed for eligibility by full text reading and 11 were excluded as per predefined criteria.

Thus 12 articles were selected for qualitative analysis [24-35] and 5 were selected from them for meta-analysis [31-35] in which denosumab was compared to bisphosphonates with outcomes as our predefined inclusion criteria. Among twelve included studies four were RCTs $[26,31,32,35]$, three were retrospective studies $[28,29,33]$, three were prospective observational studies $[24,25,34]$. Among four conferences abstracts $[27,30,33,34]$, two did not mention about study design $[27,30]$. No other studies were identified by manual search in reference lists of included studies. Figure 1 demonstrates the flowchart of details on study screening and selection.

\section{General characteristics of included studies}

Among the included studies, nine of them were from

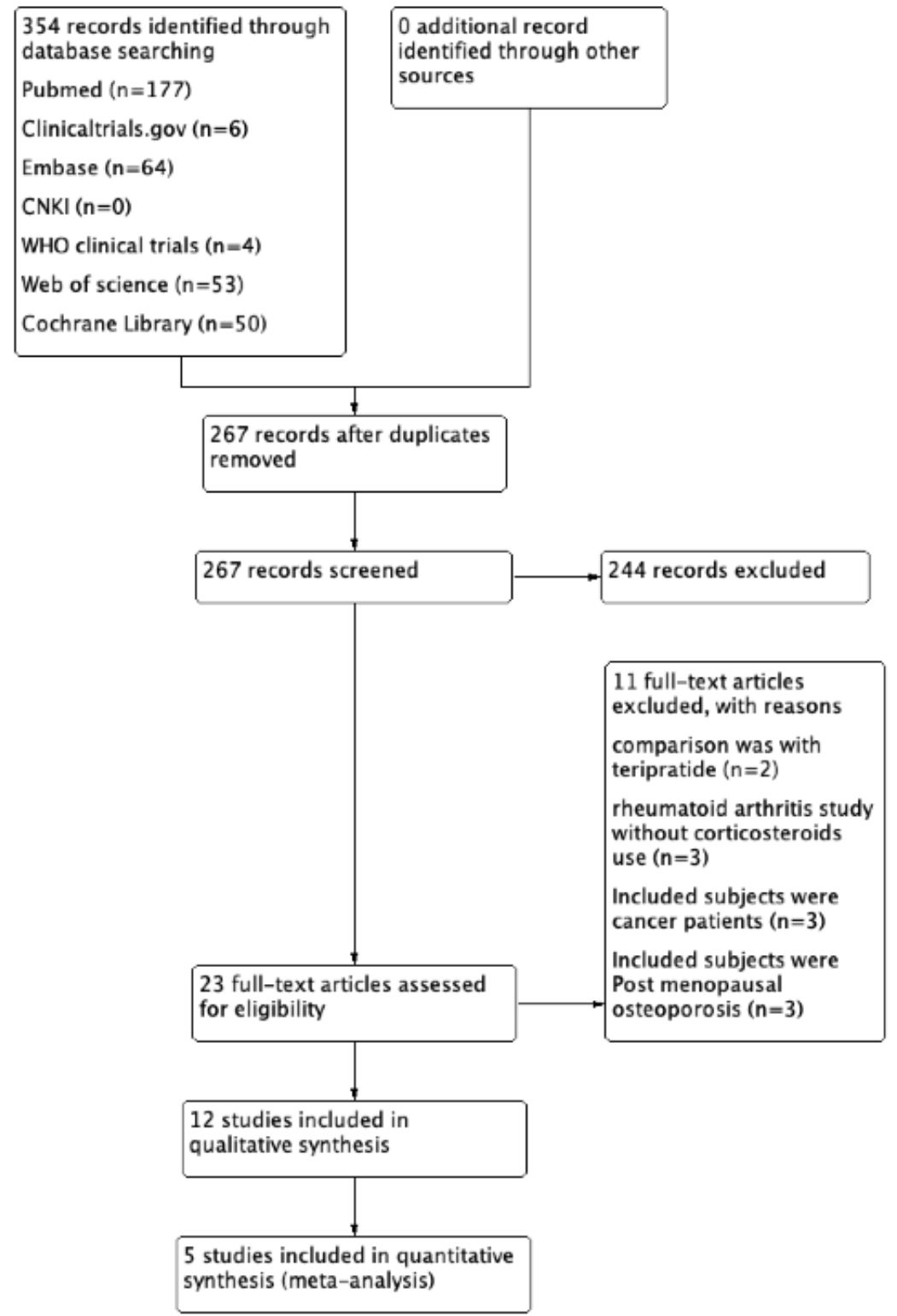

Figure 1: Flowchart of details on study screening and selection. 


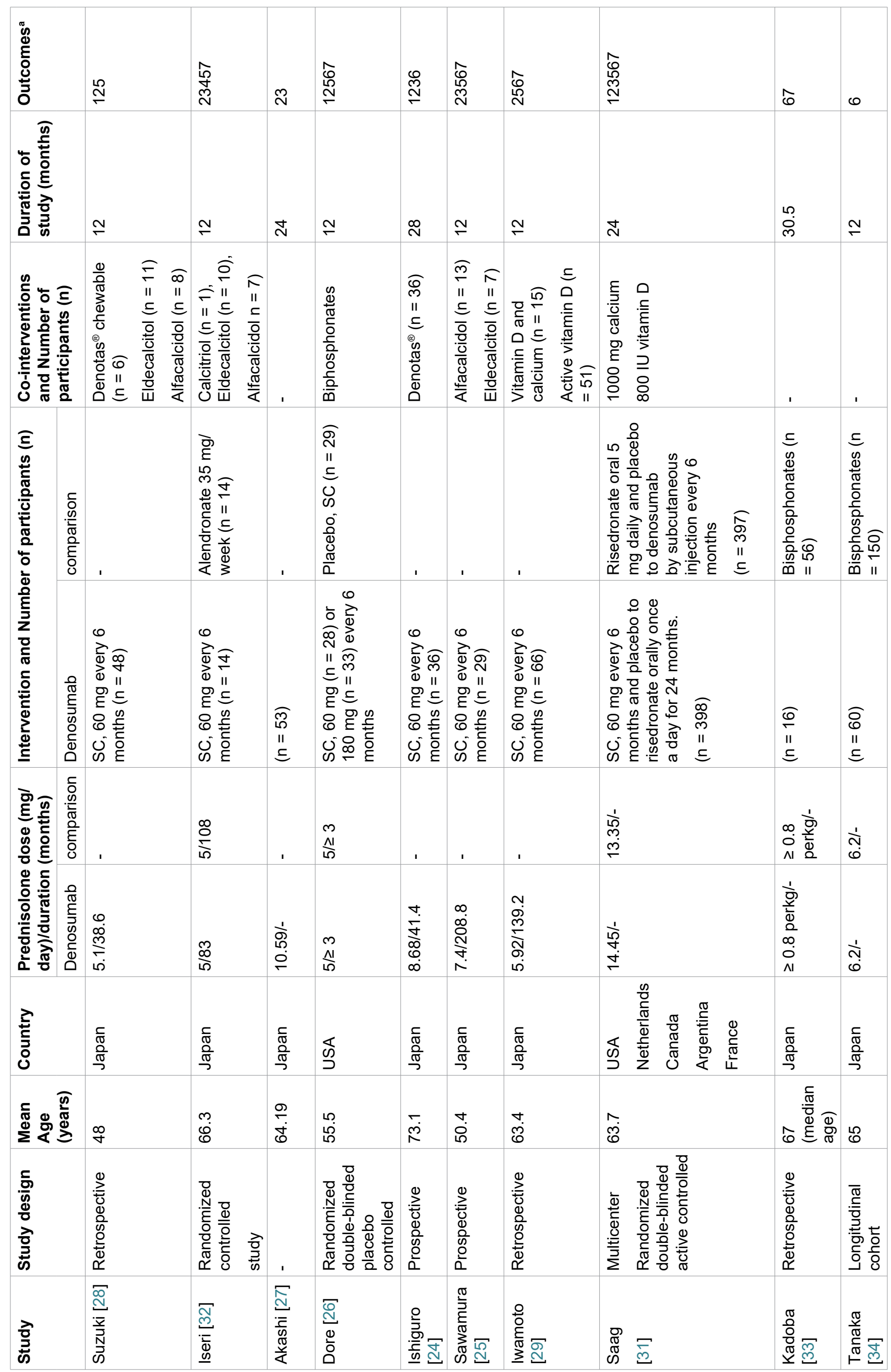




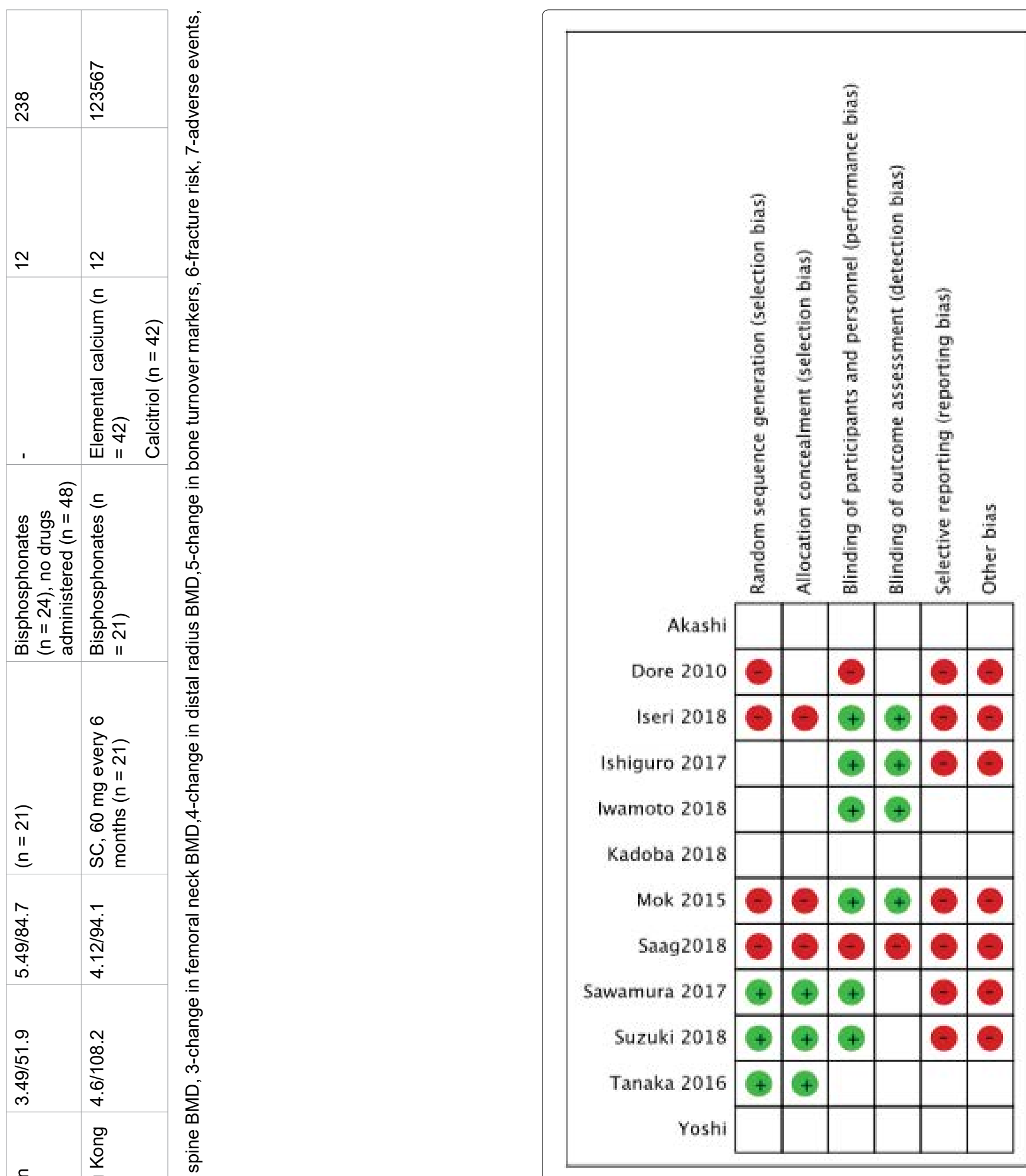

Figure 2: Risk of bias summary, - no bias, + bias, blankunclear bias.

Japan, one from the USA, one from Hong kong, and one was multicenter RCT. Publication year ranged from 2010 to 2018 . In total it included 1562 GIOP patients taking 60 $\mathrm{mg}$ of denosumab subcutaneously (SC) every 6 months or $180 \mathrm{mg} \mathrm{SC}$ every 6 months $(n=33)$ [26]. An average dose of prednisolone during the start of treatment ranged from $3.59 \mathrm{mg} /$ day to $14.45 \mathrm{mg} /$ day in denosumab group and in comparison group it ranged from $4.12 \mathrm{mg} /$ day to $13.35 \mathrm{mg} /$ day. Cointerventions used in the different study were calcium and vitamin D (Alfacalcidol, Eldecalcitol, calcitriol). Underlying diseases that caused 
the use of prednisolone in different studies were mainly rheumatic diseases, ulcerative colitis, Crohn's diseases, asthma. Further information on general characteristics of included studies is presented in Table 1.

\section{Results of bias assessment}

Four of the included studies had a low risk of bias $[26,31,32,35]$. Two studies had a high risk of bias [25,28] and the rest had an unclear risk of bias. Summary of bias assessment is presented in Figure 2.

\section{Fracture risk}

Eight studies included data on fracture risk [2426,29,31,33-35]. Among studies reported an incidence of fracture, Dore, et al. reported a fracture of less than $2 \%$ and fracture risk was not correlated to the use of denosumab, bisphosphonates, glucocorticoids. Iwamoto, et al. reported 2 fragility fractures in the pelvic ring. Studies that were included in meta-analysis revealed no statistically significant difference in fracture risk between denosumab and bisphosphonates group risk ratio $(R R)=0.62,95 \%$ Confidence interval $(\mathrm{Cl})=[0.19,1.99]$ with heterogeneity $\mathrm{I}^{2}$ of $52 \%$ (Figure 3 ).

Mok, et al. reported no fracture incidence in both treatment arms thus removed from meta-analysis. In the outcome of fracture risk, we assessed the funnel plot to evaluate publication bias, which revealed no evidence of publication bias (Figure 4). We performed sensitivity analysis to determine if overall effect size chan- ges leaving out one study at a time for the outcome of fracture risk. The result of sensitivity analysis showed the overall effect size was not changeable (Table 2). We further performed subgroup analysis on basis of risk of bias. The result of subgroup analysis revealed, studies with high/unclear risk of bias favor denosumab whereas studies with no high/unclear risk of bias favor bisphosphonates (Table 3).

\section{Adverse events}

Denosumab being relatively new drug there exists a concern of long term safety and adverse effects. Effect of denosumab on healthy Chinese man was reported by Chen, et al. which concluded denosumab was well

Table 2: Sensitivity analysis (fracture risk).

\begin{tabular}{|l|l|l|}
\hline Name of study & RR $(95 \% \mathrm{Cl})$ & $\mathrm{I}^{2}(\%)$ \\
\hline Iseri [32] & $0.49[0.13,1.89]$ & 65 \\
\hline Kadoba [33] & $0.72[0.19,2.74]$ & 63 \\
\hline Saag [31] & $0.34[0.08,1.34]$ & 16 \\
\hline Tanaka [34] & $1.10[0.65,1.85]$ & 0 \\
\hline
\end{tabular}

Table 3: Subgroup analysis based on the risk of fracture.

\begin{tabular}{|l|l|l|l|}
\hline Subgroup & $\begin{array}{l}\text { No. of included } \\
\text { studies }\end{array}$ & RR $(95 \% \mathrm{Cl})$ & $I^{2}(\%)$ \\
\hline High or unclear risk of bias \\
\hline Yes & 2 & $0.21[0.06,0.75]$ & 0 \\
\hline No & 2 & $1.16[0.68,1.98]$ & 0 \\
\hline
\end{tabular}

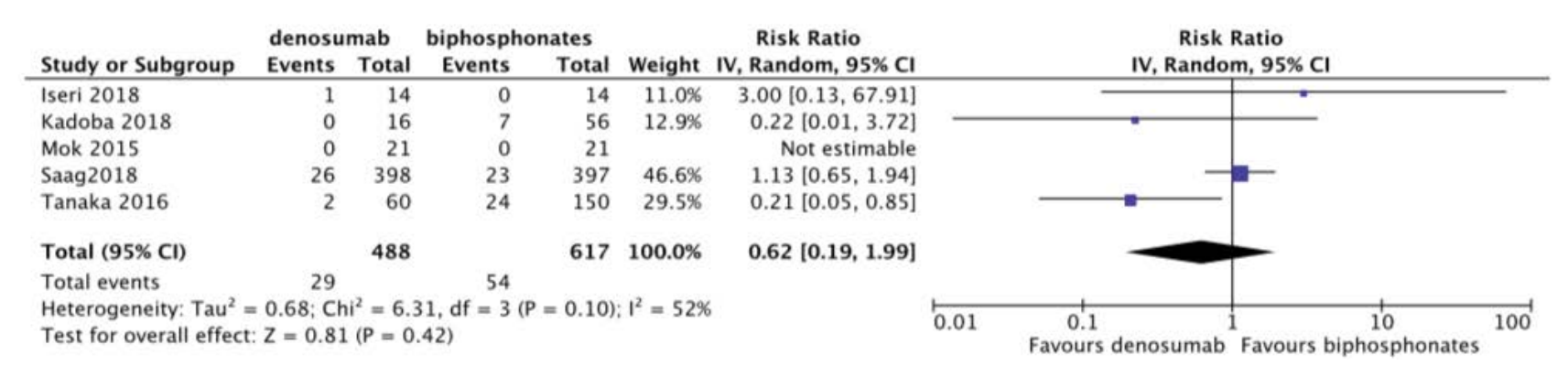

Figure 3: Forest plot of included studies comparing fracture risk.

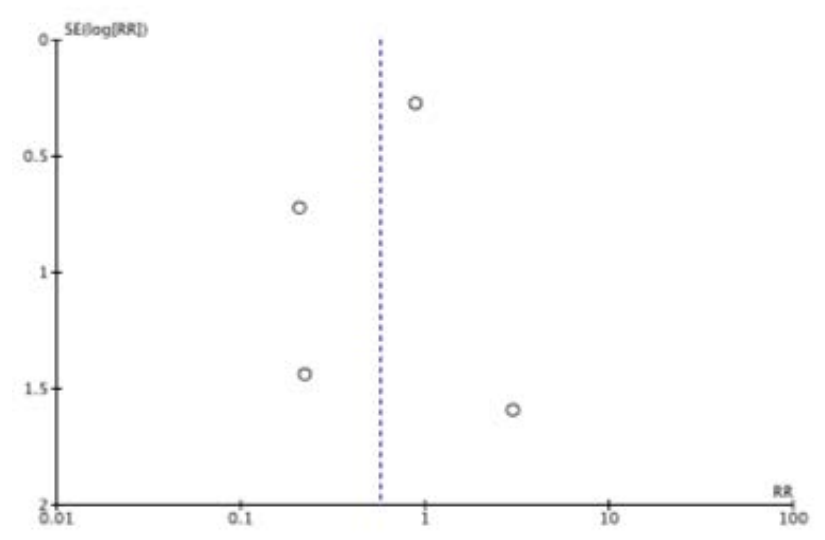

Figure 4: Funnel plot (fracture risk). 
tolerated and there was no concern about its safety [36]. Large RCTs on postmenopausal osteoporosis has revealed the safety profile of denosumab $[12,13]$. It has been reported as a useful therapeutic option for patients with renal insufficiency [37]. It has been reported that denosumab also improves glycemic control on specific group [38] and is also effective in bone marrow edema syndrome [39]. But there are some contraindication and adverse effects in use of denosumab [40]. Also reproductive toxicity [41], Alopecia areata [42] has been

Table 4: Adverse events of denosumab in GIOP.

\begin{tabular}{|c|c|c|}
\hline \multirow[t]{2}{*}{ Study } & \multicolumn{2}{|l|}{ Adverse events/reactions } \\
\hline & Denosumab & Bisphosphonates \\
\hline Suzuki 2018 & $\begin{array}{l}\text { No serious adverse event, such as hypocalcemia or } \\
\text { atypical bone fracture, occurred during the study. }\end{array}$ & N/A \\
\hline Iseri [32] & $\begin{array}{l}\text { Skin rash }(n=1) \\
\text { Pulmonary tuberculosis }(n=1) \\
\text { Hypocalcemia }(n=2) \\
\text { Femur neck fracture }(n=1)\end{array}$ & none \\
\hline Akashi [27] & Not reported & N/A \\
\hline Dore [26] & $\begin{array}{l}\text { Adverse events were comparable between } \\
\text { denosumab and placebo groups }\end{array}$ & N/A \\
\hline Ishiguro [24] & $\begin{array}{l}\text { Hypocalcemia }(n=0) \\
\text { Jaw osteonecrosis }(n=0) \\
\text { Atypical femoral fracture }(n=0)\end{array}$ & $\mathrm{N} / \mathrm{A}$ \\
\hline Sawamura [25] & Upper respiratory infections $(n=2)$ & $\mathrm{N} / \mathrm{A}$ \\
\hline Iwamoto [29] & $\begin{array}{l}\text { Herpes zoster }(n=2) \\
\text { Pneumonia }(n=1) \\
\text { Upper respiratory infection }(n=2) \\
\text { Acute gastritis }(n=1) \\
\text { Gastric ulcer }(n=1) \\
\text { Fragility fracture }(n=2) \\
\text { Colon cancer }(n=2)\end{array}$ & $\mathrm{N} / \mathrm{A}$ \\
\hline Saag [31] & $\begin{array}{l}\text { Pneumonia }(n=5) \\
\text { Cardiac failure }(n=3) \\
\text { Transient ischemic attack }(n=3) \\
\text { Atypical femoral fracture }(n=1) \\
\text { Osteoporosis related fractures }(n=26 / 398) \\
\text { Malignancy }(n=5) \\
\text { Back pain }(n=18) \\
\text { Arthralgia }(n=17) \\
\text { Hypertension }(n=15) \\
\text { Diverticulitis }(n=1) \\
\text { Pyelonephritis acute }(n=1) \\
\text { Anti-denosumab antibody }(n=1)\end{array}$ & $\begin{array}{l}\text { Pneumonia }(n=6) \\
\text { Osteoporosis related fractures }(n=23 / 397) \\
\text { Malignancy }(n=3) \\
\text { Back pain }(n=17) \\
\text { Arthralgia }(n=21) \\
\text { Hypertension }(n=13) \\
\text { Diverticulitis }(n=1) \\
\text { Pyelonephritis acute }(n=1) \\
\text { Bronchitis }(n=2)\end{array}$ \\
\hline Kadoba [33] & $\begin{array}{l}\text { Osteonecrosis of the jaw }(n=1) \\
\text { Transient hypocalcemia }(n=3)\end{array}$ & $\begin{array}{l}\text { Osteoporotic fractures }(n=7) \\
\text { Avascular necrosis of femoral head }(n=8) \\
\text { Osteonecrosis of the jaw }(n=1) \\
\text { Transient hypocalcemia }(n=6)\end{array}$ \\
\hline Tanaka [34] & Vertebral fracture $(n=2)$ & Vertebral fracture $(n=24)$ \\
\hline Yoshi [30] & Not reported & Not reported \\
\hline Mok [35] & $\begin{array}{l}\text { Fever after injection }(n=1) \\
\text { Infective episodes }(n=7) \\
\text { Dyspepsia/reflux }(n=3) \\
\text { Dizziness/vertigo }(n=2) \\
\text { High blood pressure }(n=1) \\
\text { Arthralgia }(n=1) \\
\text { Skin rash }(n=1) \\
\text { Alopecia }(n=1) \\
\text { Keratitis }(n=1)\end{array}$ & $\begin{array}{l}\text { Infective episodes }(n=1) \\
\text { High blood pressure }(n=1) \\
\text { Arthralgia }(n=1) \\
\text { Skin rash }(n=1) \\
\text { Menorrhagia }(n=1)\end{array}$ \\
\hline
\end{tabular}

N/A- not applicable. 
associated with the use of denosumab. Adverse events that were reported in the included studies are presented in Table 4. Denosumab being an antibody against RANKL, major concern in the use of denosumab is the incidence of infection.

a) Infection: Saag, et al. reported 5 cases with pneumonia in the denosumab group and 5 in risedronate group. The rate of infections between groups was similar. Mok, et al. reported 7 cases of minor upper respiratory infection which needed no antibiotics. Iseri, et al. reported a case of tuberculosis after the use of denosumab for 10 months. American College of Rheumatology (ACR) recommends denosumab as a conditional recommendation for GIOP due to lack of safety data in people treated with immunosuppressive agents. Three of the included studies reported data of adverse effects on concomitant use of immunosuppressive agents $[26,29,31]$. Iwamoto, et al. reported 4 out of 5 patients treated with immunosuppressive suffered from infections. Saag, et al. reported the incidence of infection was similar in patients with or without immunosuppressive agents (biologic or non-biologic). Dore, et al. reported the use of immunosuppressive agents in 8 patients and adverse events were comparable between denosumab and placebo groups. It has also been reported that the incidence of serious and opportunistic infections treated with denosumab and biologics for rheumatoid arthritis were low [43].

b) Eczema: Eczema resulted in withdrawal from a study for 1 patient but it got better without any treatment after withdrawal from study [32]. Other skin related adverse effects reported were skin rash and alopecia.

c) Hypocalcemia: Vitamin D and calcium use are essential during treatment with denosumab $[44,45]$. Calcium and vitamin $D$ were used as cointervention in many studies included in this review (Table 1). About the incidence of hypocalcemia during the treatment Iseri, et al. reported 2 cases in the denosumab group, Kadoba, et al. reported 3 cases in denosumab and 6 cases in bisphosphonates group. Further hypocalcemia is a contraindication for denosumab use, calcium and vitamin $D$ level should be checked regularly during the treatment period. Iseri, et al. and Saag, et al. excluded patients with low calcium and vitamin $D$ level from their study. d) Osteonecrosis of Jaw (ONJ): Bad dental hygiene, dental extraction, use of chemotherapy, dental prosthetics are risk factors to develop osteonecrosis of jaw [46]. Denosumab being a potent antiresorptive agent there is a possibility of incidence of ONJ. Kadoba, et al. reported 1 case each in denosumab and bisphosphonates group. 10 years extension of FREEDOM trails reported 13 cases of ONJ consistent with 5.2 per 10,000 participant-years [13].

Adverse events frequently reported with use of denosumab were e) Atypical fracture of femur f) Back pain g) Arthralgia. In the included studies other adverse events associated with denosumab use were cardiac failure, transient ischemic attack, hypertension, anti-denosumab antibodies [31] as presented in Table 4. Hepatic toxicity has also been reported with use of Denosumab [47].

We performed Meta-analysis comparing the incidence of adverse events between denosumab and bisphosphonates. There was no significant difference in the incidence of adverse events between denosumab and bisphosphonates $\mathrm{RR}=1.67,95 \% \mathrm{Cl}=[0.75,3.75]$ with heterogeneity $\mathrm{I}^{2}$ of $75 \%$ (Figure 5 ).

\section{Change in BMD}

Current knowledge of denosumab confirms positive effect in increasing BMD $[13,14]$. Further, it has been reported to be superior to bisphosphonates [11] in treating post-menopausal osteoporosis. Iwamoto, et al., Mok, et al., Iseri, et al., Saag, et al. reported change in lumbar BMD by $4.4 \%$, greater than $3 \%, 5.3 \%, 4.1 \%$ respectively. Change in BMD was higher in GC continuing group than GC starting group as reported by Saag, et al. which could be probably because of a higher dose of GC in GC starting group. Further, Saag, et al. reported denosumab not only to be non-inferior but superior to risedronate.

One of the studies reported the change in distal radius BMD but there was no significant change in BMD after use of denosumab for 12 months [32]. Another study where first 6 months patients were treated with either denosumab or bisphosphonates then changed to other agent showed those who were treated with bisphosphonates then changed to denosumab after 6 months had their BMD increased in lumbar spine, fe-

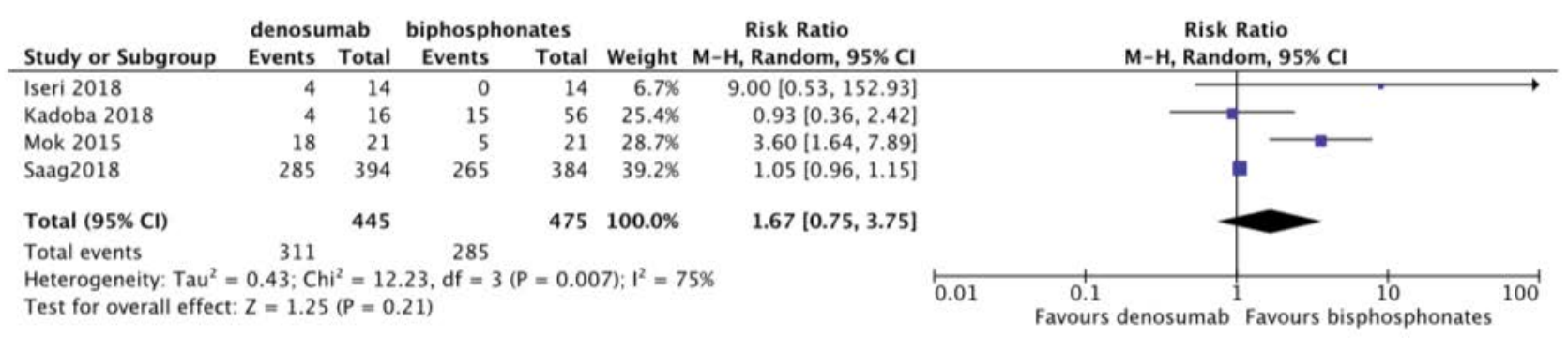

Figure 5: Forest plot of included studies comparing adverse events. 
mur neck and greater trochanter compared with baseline. But switching from denosumab to bisphosphonates after 6 months decreased greater trochanter BMD but there was increased in BMD in other sites [30]. Iwamoto, et al. reported patients who transitioned from bisphosphonates showed an increment of $4.17 \%$ and from teriparatide showed an increment of $3.71 \%$ in overall BMD after 12 months [29]. Similarly, Suzuki, et al. reported $4.2 \%$ of increment in lumbar BMD and $4.7 \%$ of increment in total hip BMD in bisphosphonates pretreated group [28]. Iwamoto, et al. reported multivariate logistic analysis which revealed a dose of prednisolone and body weight were associated with the change in greater than $3 \%$ BMD.

Meta-analysis was done to compare the efficacy of denosumab to bisphosphonates in change in BMD. There was no statistically significant difference for change in femoral neck BMD MD $=1.0295 \% \mathrm{Cl}=[-0.76,2.80] \mathrm{I}^{2}$ $=98 \%$ (Figure 6 ). Change in total hip and lumbar spine $\mathrm{BMD}$ were significant $\mathrm{MD}=1.0395 \% \mathrm{Cl}=[0.12,1.94] \mathrm{I}^{2}$ $=91 \%$ (Figure 7 ) and $\mathrm{MD}=2.4895 \% \mathrm{Cl}=[1.74,3.23] \mathrm{I}^{2}=$ $78 \%$ (Figure 8) respectively. Change in BMD in the distal radius and greater trochanter were not analyzed as it was reported in one study only.

\section{Change in bone turnover markers}

Seven studies reported the change in bone turnover markers [25,26,28,29,31,32,35]. Change in level of procollagen type $1 \mathrm{~N}$-terminal propeptide (P1NP), osteocalcin, bone alkaline phosphatase (BAP) were measured as bone formation markers, whereas tartrate-resistant acid phosphatase-5b, type I collagen cross-linked C-telopeptide (CTX) were measured as markers of bone resorption. Use of denosumab resulted in a rapid decrease in bone turnover markers in included studies. Mok, et al. reported marked decreased in bone turnover markers in patients that were switched from bisphosphonates to denosumab than who continued bisphosphonates at 12 months. Suzuki, et al. and Iseri, et al. reported marked decrease in both denosumab and bisphosphonates groups, but between these groups there was no significant difference. Saag, et al. reported marked reduced in CTX in the $10^{\text {th }}$ day of denosumab which makes it a better option for GIOP as GC induce rapid loss of BMD in the first few months. Dore, et al. reported baseline P1NP correlated with BMD increment for lumbar spine and total hip. They also reported CTX and P1NP levels increased at the end of each 6 months dosing interval pointing requirement of next dose.

\section{Discussion}

Our systemic review and meta-analysis reviewed all available studies on the efficacy and safety of denosumab in GIOP. We found that denosumab increased BMD

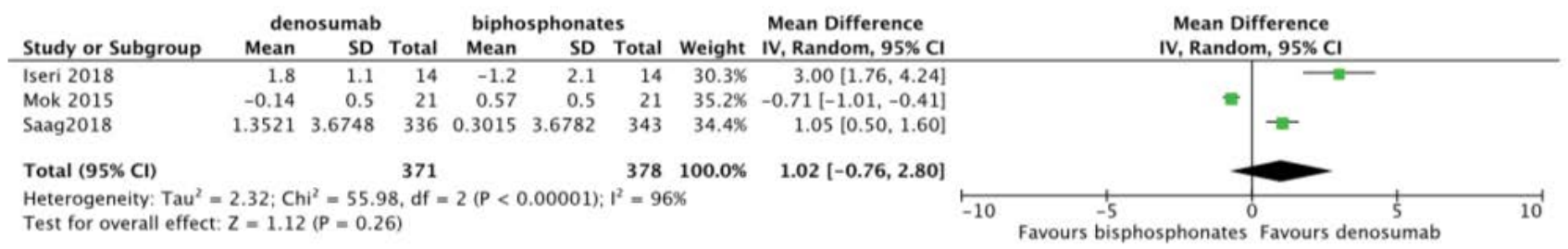

Figure 6: Forest plot of included studies comparing femoral neck BMD.

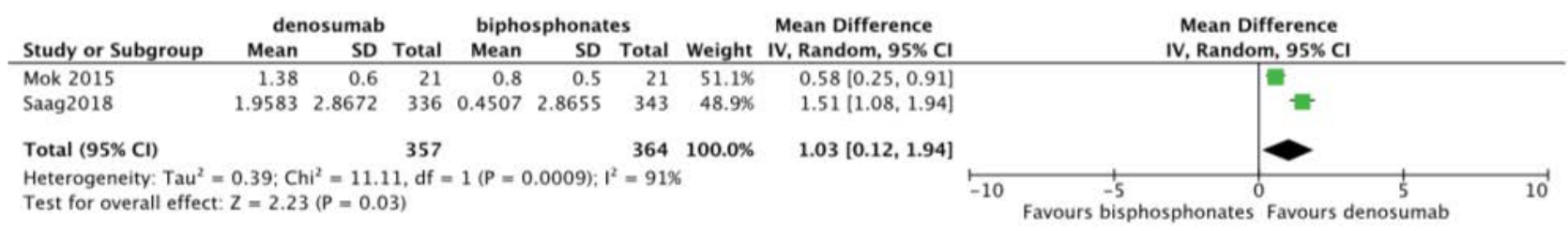

Figure 7: Forest plot of included studies comparing total hip BMD.

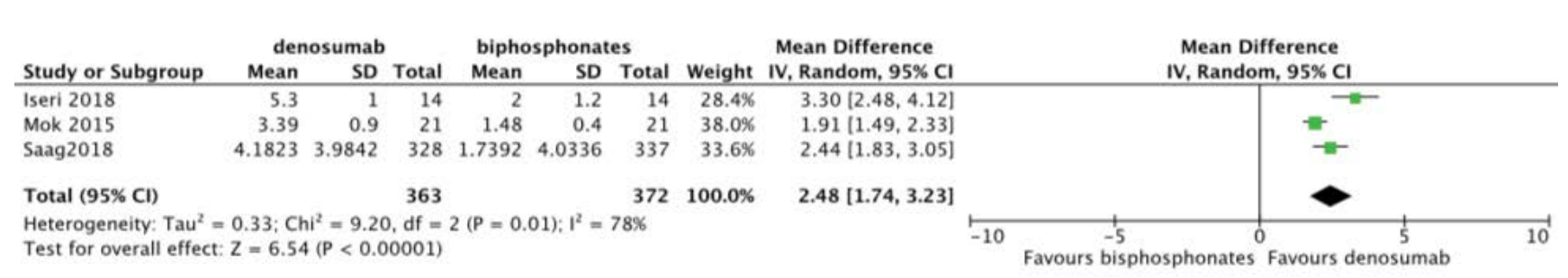

Figure 8: Forest plot of included studies comparing lumbar spine BMD. 
in different bone sites, decreased bone turnover markers but there was no significant difference in fracture risk and adverse events when compared with bisphosphonates. Although there was a gain in BMD, which didn't correlate with fracture risk benefit. This could be because of the short follow up period in the included studies or these points out multifactorial association with fracture incidence [48]. Our findings were similar to meta-analysis done on post-menopausal osteoporosis for the efficacy and safety of denosumab compared with bisphosphonates [14].

There were only 1 large RCT and 2 small RCTs available on this topic. To our knowledge, this is the first meta-analysis done on this topic which quantifies all possible outcomes data available in the literature. To increase comprehensiveness and to balance the picture on the efficacy of denosumab we included grey literature too, which also helped to reduce publication bias. We included 2 conference abstracts in meta-analysis. Thus, we did a subgroup analysis based on a risk of bias for fracture risk, which resulted studies with high/unclear risk of bias favor denosumab whereas studies with no high/unclear risk of bias favor bisphosphonates. There was significant heterogeneity in this meta-analysis which could be because there were few studies in this meta-analysis and also the fact we considered including RCTs and observational studies together [49]. Although there is no meta-analysis on this topic, a systemic review has been published [50]. Coskun reported RCTs and observational studies but didn't include conference abstracts. Coskun also added 2 studies which included post-menopausal osteoporosis patients on GC therapy which we didn't include in our study [51,52].

Dore, et al. recruited 29 patients who took both denosumab and bisphosphonates which give us insight on how the outcome would be after switching from bisphosphonates to denosumab because bisphosphonates effect persists after discontinuation too. They reported a marked decrease in bone turnover markers and had greater increased in BMD. Switching from one osteoporosis agent to denosumab was made in studies conducted by Ishiguro, et al. [24], Suzuki, et al. [28], Iwamoto, et al. [29], Mok, et al. [35], Iseri, et al. [32], all of these studies reported continuous increased in BMD after switching.

ACR recommends denosumab as a conditional recommendation for GIOP due to lack of data in concomitant use with immunosuppressants. In our study, we found out that there was no significant difference in adverse events between denosumab and bisphosphonates groups. Further, studies that included concomitant use of immunosuppressants also reported no significant difference in adverse events. A major concern with denosumab is an infection, as immune cells also express RANK. But some studies suggested denosumab has no effect on RANKL of immune system [53,54] suggesting it to be safe in terms of infection incidence. ACR also re- commends teriparatide as a conditional recommendation for GIOP but to our knowledge, there is no available head to head trials comparing denosumab and teriparatide in GIOP. ACR first choice for GIOP, bisphosphonates might not be ideal for women with child bearing potential as bisphosphonates have a strong affinity to the bone and stay in bone for years even after discontinuation, this might be a concern in young female population who commonly have an autoimmune disease requiring long term $\mathrm{GC}$.

Further, for real world adherence of drugs, not only efficacy and safety but cost effectiveness of drugs also should be evaluated. We searched for studies which compared denosumab with other available options for osteoporosis in terms of cost effectiveness. Hiligsmann, et al. and Yoshizawa, et al. reported denosumab to be cost effective than other options for osteoporosis $[55,56]$. Study on drug compliance also reported denosumab has higher compliance [57].

Our study had limitations, the number of studies were few and there was only 1 large RCT. Duration of follow-up in all included studies was short thus change in BMD didn't correlate with fracture risk benefits and long-term adverse effects were underestimated. Most of the studies were carried out in Asia so it might be hard to estimate safety and tolerability in other groups of the population. Multicenter large RCT by Saag, et al. was funded by a pharmaceutical company which might increase performance bias. None of the included studies reported using zoledronic acid which is one of the most potent bisphosphonates. But it is worth to mention that in post-menopausal osteoporosis denosumab was found more effective in increasing BMD and decreasing bone turnover markers than zoledronic acid [58].

\section{Conclusions}

As per currently available evidence, denosumab is effective in increasing BMD with no difference in adverse events when compared to bisphosphonates. Although denosumab has some limitations, it also has benefits of cost effectiveness and drug adherence when compared to bisphosphonates. But only a few studies are currently available thus more large RCT with long term follow up and with the primary outcome as fracture are needed. Also, studies assessing the safety of denosumab in women of childbearing potential and fracture risk in GC treated children are needed. Further, we should emphasize the need for primary and secondary prevention of fracture in patients using GC.

\section{Author's Contributions}

AS and SX created the study design. HIR and JJ searched and screened the titles in databases. AS and MMEA drafted the article. All authors read and approved the final draft. 


\section{Declarations of Interest}

None.

\section{Founding Source}

This research did not receive any specific grant from funding agencies in the public, commercial, or not-forprofit sectors.

\section{References}

1. Rosen CJ, Bouillon R, Compston JE, Rosen V (2013) Primer on the metabolic bone diseases and disorders of mineral metabolism. ( $8^{\text {th }}$ edn), John Wiley \& Sons, Ames, USA.

2. Angeli A, Guglielmi G, Dovio A, Capelli G, de Feo D, et al (2006) High prevalence of asymptomatic vertebral fractures in post-menopausal women receiving chronic glucocorticoid therapy: A cross-sectional outpatient study. Bone 39: 253-259.

3. Curtis JR, Westfall AO, Allison J, Bijlsma JW, Freeman A, et al. (2006) Population-based assessment of adverse events associated with long-term glucocorticoid use. Arthritis Rheum 55: 420-426.

4. Kaji H, Yamauchi M, Chihara K, Sugimoto T (2006) The threshold of bone mineral density for vertebral fracture in female patients with glucocorticoid-induced osteoporosis. Endocr J 53: 27-34.

5. LoCascio V, Bonucci E, Imbimbo B, Ballanti P, Adami S, et al. (1990) Bone loss in response to long-term glucocorticoid therapy. Bone Miner 8: 39-51.

6. Feldstein AC, Elmer PJ, Nichols GA, Herson M (2005) Practice patterns in patients at risk for glucocorticoid-induced osteoporosis. Osteoporos Int 16: 2168-2174.

7. Buckley L, Guyatt G, Fink HA, Cannon M, Grossman J, et al. (2017) 2017 American College of Rheumatology guideline for the prevention and treatment of glucocorticoid-induced osteoporosis. Arthritis Rheumatol 69: 1521-1537.

8. McNicholl DM, Heaney LG (2010) The safety of bisphosphonate use in pre-menopausal women on corticosteroids. Curr Drug Saf 5: 182-187.

9. Marini JC (2003) Do bisphosphonates make children's bones better or brittle? N Engl J Med 349: 423-426.

10. Park JH, Park EK, Koo DW, Lee S, Lee SH, et al. (2017) Compliance and persistence with oral bisphosphonates for the treatment of osteoporosis in female patients with rheumatoid arthritis. BMC Musculoskelet Disord 18: 152.

11. Brown JP, Prince RL, Deal C, Recker RR, Kiel DP, et al. (2009) Comparison of the effect of denosumab and alendronate on BMD and biochemical markers of bone turnover in postmenopausal women with low bone mass: A randomized, blinded, phase 3 trial. J Bone Miner Res 24: 153-161.

12. Cummings SR, Martin JS, McClung MR, Siris ES, Eastell $\mathrm{R}$, et al. (2009) Denosumab for prevention of fractures in postmenopausal women with osteoporosis. $\mathrm{N}$ Engl $\mathrm{J} \mathrm{Med}$ 361: 756-765.

13. Bone HG, Wagman RB, Brandi ML, Brown JP, Chapurlat R, et al. (2017) 10 years of denosumab treatment in postmenopausal women with osteoporosis: results from the phase 3 randomised FREEDOM trial and open-label extension. Lancet Diabetes Endocrinol 5: 513-523.

14. Beaudoin C, Jean S, Bessette L, Ste-Marie LG, Moore L, et al. (2016) Denosumab compared to other treatments to prevent or treat osteoporosis in individuals at risk of fractu- re: A systematic review and meta-analysis. Osteoporos Int 27: 2835-2844.

15. Camacho PM, Petak SM, Binkley N, Clarke BL, Harris ST, et al. (2016) American Association of Clinical Endocrinologists and American College Of Endocrinology clinical practice guidelines for the diagnosis and treatment of postmenopausal osteoporosis - 2016. Endocr Pract 22: 11111118.

16. Jabbar S, Drury J, Fordham JN, Datta HK, Francis RM, et al. (2011) Osteoprotegerin, RANKL and bone turnover in postmenopausal osteoporosis. J Clin Pathol 64: 354-357.

17. Ferrari S, Ringe JD (2010) Pathophysiology of postmenopausal osteoporosis. In: Rizzoli R, Atlas of Postmenopausal Osteoporosis. Springer Healthcare Ltd, Tarporley, 1-32.

18. Sivagurunathan S, Muir MM, Brennan TC, Seale JP, Mason RS, et al. (2005) Influence of glucocorticoids on human osteoclast generation and activity. J Bone Miner Res 20: 390-398.

19. Compston J (2010) Management of glucocorticoid-induced osteoporosis. Nat Rev Rheumatol 6: 82-88.

20. Brown JP, Dempster DW, Ding B, Dent-Acosta R, Martin JS, et al. (2011) Bone remodeling in postmenopausal women who discontinued denosumab treatment: Off-treatment biopsy study 26: 2737-2744.

21. https://www.accessdata.fda.gov/drugsatfda_docs/label/2018/125320s186lbl.pdf

22. Moher D, Liberati A, Tetzlaff J, Altman DG, PRISMA Group (2009) Preferred reporting items for systematic reviews and meta-analyses: The PRISMA statement. PLoS Med 6: e1000097.

23. Higgins JPT, Green S (2011) Cochrane handbook for systematic reviews of interventions version 5.1.0.

24. Ishiguro S, Ito K, Nakagawa S, Hataji O, Sudo A (2017) The clinical benefits of denosumab for prophylaxis of steroid-induced osteoporosis in patients with pulmonary disease. Arch Osteoporos 12: 44.

25. Sawamura M, Komatsuda A, Togashi M, Wakui H, Takahashi N, et al. (2017) Effects of denosumab on bone metabolic markers and bone mineral density in patients treated with glucocorticoids. Intern Med 56: 631-636.

26. Dore RK, Cohen SB, Lane NE, Palmer W, Shergy W, et al. (2010) Effects of denosumab on bone mineral density and bone turnover in patients with rheumatoid arthritis receiving concurrent glucocorticoids or bisphosphonates. Ann Rheum Dis 69: 872-875.

27. Akashi K, Nishimura K, Kageyama G, Ichikawa S, Shirai T, et al. (2017) FRI0553 The efficacy of 2-years denosumab treatment for glucocorticoid-induced osteoporosis (GIOP). Annals of the Rheumatic Diseases 76: 699.

28. Suzuki T, Nakamura Y, Kato H (2018) Significant improvement of bone mineral density by denosumab without bisphosphonate pre-treatment in glucocorticoid-induced osteoporosis. Mod Rheumatol 28: 885-889.

29. Iwamoto N, Ueki Y, Aramaki T, Ichinose K, Eguchi K, et al. (2018) THU0494 The efficacy of denosumab in glucocorticoid-induced osteoporosis did not depend on prior treatment but was affected by the dosage of glucocorticoid. Annals of the Rheumatic Diseases 77: 454.

30. Yoshii I, Chijiwa T (2017) Denosumab as a first choice drug for glucocorticoid induced osteoporosis treatment instead of bisphosphonate. Annals of the Rheumatic Diseases 76: 1349. 
31. Saag KG, Wagman RB, Geusens P, Adachi JD, Messina OD, et al. (2018) Denosumab versus risedronate in glucocorticoid-induced osteoporosis: A multicentre, randomised, double-blind, active-controlled, double-dummy, non-inferiority study. Lancet Diabetes Endocrinol 6: 445-454.

32. Iseri $K$, lyoda $M$, Watanabe $M$, Matsumoto $K$, Sanada $D$, et al. (2018) The effects of denosumab and alendronate on glucocorticoid-induced osteoporosis in patients with glomerular disease: A randomized, controlled trial. PLoS One 13: e0193846.

33. Kadoba K, Nishimura K, Mukoyama H, Saito R, Waki D, et al. (2018) The efficacy of denosumab for prevention of osteoporotic fractures in patients with connective tissue diseases receiving very high doses of glucocorticoid. Arthritis Rheumatol 70: 2.

34. Tanaka I, Ushikubo M, Izumi K, Akiya K, Oshima H (2016) A longitudinal cohort study of denosmab and bisphosphonate for prevention of vertebral fracture in glucocorticoid-induced osteoporosis in Japanese. Arthritis Rheumatol 68 : 450.

35. Mok CC, Ho LY, Ma KM (2015) Switching of oral bisphosphonates to denosumab in chronic glucocorticoid users: A 12-month randomized controlled trial. Bone 75: 222-228.

36. Chen Q, Hu C, Liu Y, Song R, Zhu W, et al. (2018) Pharmacokinetics, pharmacodynamics, safety, and tolerability of single-dose denosumab in healthy Chinese volunteers: A randomized, single-blind, placebo-controlled study. PLoS One 13: e0197984.

37. Block GA, Bone HG, Fang L, Lee E, Padhi D (2012) A single-dose study of denosumab in patients with various degrees of renal impairment. J Bone Miner Res 27: 14711479.

38. Chew CK (2017) Denosumab improves glycemic control of type 2 diabetic or prediabetic patients with osteoporosis. Journal of Bone and Mineral Research 32: S107-S107.

39. Rolvien T, Schmidt T, Butscheidt S, Amling M, Barvencik $F(2017)$ Denosumab is effective in the treatment of bone marrow oedema syndrome. Injury 48: 874-879.

40. https://dailymed.nlm.nih.gov/dailymed/druglnfo.cfm?seti$\mathrm{d}=49 \mathrm{e} 5 \mathrm{afe} 9-\mathrm{a0} \mathrm{c} 7-40 \mathrm{c} 4-\mathrm{af} 9 \mathrm{f}-\mathrm{f} 287 \mathrm{a} 80 \mathrm{c} 5 \mathrm{c} 88$

41. Bussiere JL, Pyrah I, Boyce R, Branstetter D, Loomis M, et al. (2013) Reproductive toxicity of denosumab in cynomolgus monkeys. Reprod Toxicol 42: 27-40.

42. Lyakhovitsky A, Oshinsky S, Gilboa S, Barzilai A (2016) Alopecia areata after denosumab treatment for osteoporosis. JAAD Case Rep 2: 298-300.

43. Lau AN, Wong-Pack M, Rodjanapiches R, loannidis G, Wade S, et al. (2018) Occurrence of serious infection in patients with rheumatoid arthritis treated with biologics and denosumab observed in a clinical setting. J Rheumatol 45: 170-176.

44. Suzuki T, Nakamura Y, Kato H (2018) Vitamin D and calcium addition during denosumab therapy over a period of four years significantly improves lumbar bone mineral density in Japanese osteoporosis patients. Nutrients 10: 272.

45. Nakamura $Y$, Suzuki T, Kamimura M, Murakami K, Ikegami $S$, et al. (2017) Vitamin D and calcium are required at the time of denosumab administration during osteoporosis treatment. Bone Res 5: 17021.

46. Boquete-Castro A, Gómez-Moreno G, Calvo-Guirado JL, Aguilar-Salvatierra A, Delgado-Ruiz RA (2016) denosumab and osteonecrosis of the jaw. A systematic analysis of events reported in clinical trials. Clin Oral Implants Res 27: 367-375.

47. Malnick S, Maor Y, Melzer E, Ziv-Sokolowskaia NN, Neuman MG (2017) Severe hepatocytotoxicity linked to denosumab. Eur Rev Med Pharmacol Sci 21: 78-85.

48. Hayashi K, Yamamoto M, Murakawa Y, Yamauchi M, Kaji $\mathrm{H}$, et al. (2009) Bone fragility in male glucocorticoid-induced osteoporosis is not defined by bone mineral density. Osteoporos Int 20: 1889-1894.

49. von Hippel PT (2015) The heterogeneity statistic I(2) can be biased in small meta-analyses. BMC Med Res Methodol 15: 35.

50. Coskun Benlidayi I (2018) Denosumab in the treatment of glucocorticoid-induced osteoporosis. Rheumatol Int 38: 1975-1984.

51. Matsuno H (2016) Assessment of distal radius bone mineral density in osteoporosis patients receiving denosumab, including those with rheumatoid arthritis and those receiving oral glucocorticoids. Drugs R D 16: 347-353.

52. Petranova T, Sheytanov I, Monov S, Nestorova R, Rashkov $R$, et al. (2014) Denosumab improves bone mineral density and microarchitecture and reduces bone pain in women with osteoporosis with and without glucocorticoid treatment. Biotechnol Biotechnol Equip 28: 1127-1137.

53. Stolina M, Kostenuik PJ, Dougall WC, Fitzpatrick LA, Zack DJ (2007) RANKL inhibition: From mice to men (and women). Adv Exp Med Biol 602: 143-150.

54. Bekker PJ, Holloway DL, Rasmussen AS, Murphy R, Martin SW, et al. (2004) A single-dose placebo-controlled study of AMG 162, a fully human monoclonal antibody to RANKL, in postmenopausal women. J Bone Miner Res 19: 1059-1066.

55. Hiligsmann M, Reginster JY (2011) Cost effectiveness of denosumab compared with oral bisphosphonates in the treatment of post-menopausal osteoporotic women in Belgium. Pharmacoeconomics 29: 895-911.

56. Yoshizawa T, Nishino T, Okubo I, Yamazaki M (2018) Cost-effectiveness analysis of drugs for osteoporosis treatment in elderly Japanese women at high risk of fragility fractures: Comparison of denosumab and weekly alendronate. Arch Osteoporos 13: 94.

57. Morizio P, Burkhart JI, Ozawa S (2018) Denosumab: A unique perspective on adherence and cost-effectiveness compared with oral bisphosphonates in osteoporosis patients. Ann Pharmacother 52: 1031-1041.

58. Miller PD, Pannacciulli N, Brown JP, Czerwinski E, Nedergaard BS, et al. (2016) Denosumab or Zoledronic acid in postmenopausal women with osteoporosis previously treated with oral bisphosphonates. J Clin Endocrinol Metab 101: 3163-3170. 